the contents of the packets ; and on whether the two layers of filter paper are sufficient to prevent the entrance of microorganisms. These packages are sterilized by superheated steam in the disinfecting oven of the hospital, dried in the same oven, and kept in a large dry box (the practitioner keeps his gauze in a dry box or chest). The outer filter paper may be quite dark from dust; it matters not; so long as no water comes in contact with the outer layer of the filter paper, it is absolutely certain that the inner layer of the filter paper and the contents of the packet-gauze and wool-are sterile.

You see here a number of cultures obtained by the usual methods. In each tube you will observe the same growth. They consist of fungi and micro-organisms, and the tubes were inoculated from the outer layer of the filter paper. The tubes in which I have placed portions of the inner layer of filter paper and of gauze and of cotton wool are quite sterile. Thus we can be sure that the contents of the package are sterile. How long do they remain sterile? This is important for the country practitioner to know, who perhaps one night might have to undertake an amputation and take his packages with him. He then cannot waste time by boiling his dressings. The oldest package I have tested was nine years old. Its contents were sterile. Can one then travel with the packages without risking their sterile properties? I have often done so, and on my return home have found their contents sterile, even though they had lain together in my handbag with the usual travelling requisites. The longest journeys have been from Copenhagen to the Equator, and back again. The contents were still sterile. So the packages can be employed with safety, and when the thick layer of dressing is wetted with carbolic solution, it is also antiseptic.

Gentlemen, our patient is now dressed, and thanks to Lister one has good reason to hope for a useful arm, because the dressing which is applied, and which gives him the hope of recovery, is Lister's. When I first made a communication as to this dressing and these packages, at the Fourth 'Surgical Congress, in Paris, in I889, I called it " a safe and practical modification of Lister's dressing." And although one can name Hippocrates because he has said that wounds should be treated dry, and although one can name Chassaignac because he has taught us how important it is to drain well, you will not for a moment doubt that had. Lister not existed neither would there have been such good prospects for our patient.

Lister's invention is the greatest that jas been made within the domain of surgery. Lister's only fault is that he has created so many operators who are not surgeons, because it is quite clear since one can do a great deal unpunished, thanks to antiseptics, that it does not certainly follow that there are indications to do it.

Look at our patient again. As I have said to you, before Lister's day this working man would have lost his right arm after 'such an accident. Now he keeps it. Thanks to conservatism! Thanks to antisepticism! Thanks to Lister !

\section{A (Iontribution}

\author{
TO THE
}

\section{TREATMENT OF FRACTURED PATELLA.}

\section{By Professor VON MIKULICZ-RADECKI,}

Professor of Surgery at the University of Breslau.

A Tribute to Lord Lister.

WHEN I was invited by the editorial staff of the British Medical Journal to take part in the celebration in honour of Lord Lister by a contribution, I accepted the invitation with great pleasure, for it was not only an honourable task to show my esteem in this way for a universally revered master, but it procured for me the great satisfaction of expressing my personal thanks to the man who had first given surgeons a joy in their profession. When, however, I set out to write an article with this end in view in accordance with the wish of the Editor, I fell into no inconsiderable difficulties. What sort of a subject should I choose?. Where should I begin and where end? The whole development of surgery in the last twenty-five years is one long hymn in honour of Joseph Lister, and can a page of the history of modern surgery exist without reference to the inventor of the present method of the treatment of wounds? He who would worthily celebrate Lister to-day must write a whole book taking into consideration gynaecology, obstetrics, pathological anatomy, and bacteriology; in short, most of the disciplines of medicine as well as surgery. So I must confine myself to taking one little leaf from Lister's laurel wreath as the point of departure of my essay.

And yet the temptation to go beyond it was for me very great. Have I not from the outset risen up and fought for the development of modern surgery? For it was, indeed, a battle for young surgeons twenty-five years ago to bring into acceptation the variously assailed teachings of Lister. I still have a lively recollection of my student days in which the various kinds of wound infection in the shape of the so called accidental traumatic diseases-erysipelas, pyaemia, and wound diphtheria-filled the surgical wards with horror. Often for months all surgical operations were deferred because wound infections could not be subdued. At the same time I saw with my own eyes surgical operations practised a few hours later on the cadaver on the same table on which a living person had been operated on in the morning, and the same sponges and instruments not at all or only imperfectly cleaned, used for both purposes. At that time they washed well after the opera. tion in order to clean the blood-stained hands, but before the operation this was a superfluous luxury. This I saw as a student.

When, in the year 1875 , I became assistant to Billroth, the open treatment of wounde still prevailed at his hospital, by which, considering the circumstances at the time, relatively good results were obtained - that is, better than with many other surgeons, and these were certainly few enough. He was delighted if the wound left by an amputation of the breast healed by granulation in two to three months. Every operation in which the wound had to be elosed was dangerous ; even with a simple so-called subcutaneous osteotomy fever and pyaemia occasionally occurred. My teacher, Billroth, who, as was well known, had occupied himself for years with the problem of the treatment of wounds, and had contributed many important explanations of the question, was strongly urged by his friend, Volkmann, to accept Lister's principles. It was not easy for Billroth to give way absolutely to this compulsion. Any one who was acquainted with this able man would easily understand how difficult it was for him to adopt a procedure which only promised good results to a most painstaking, almost slavish, imitation, and the principles of which were not in agreement with his views on traumatic diseases at that time.

In spite of this inward opposition Billroth was eagerly bent. on introducing the Listerian treatment of wounds into his hospital. With this object he sent one assistant after another to learn about the new procedure at the source; so first Gussenbauer, then Wölfler, and lastly I (in the year 1879) had the opportunity of learning to know through our own observations Lister's technique and method. The stay at King's College Hospital is one of the happiest recollections of $\mathbf{m y}$ travelling days as a student at that time. Lister was never tired of showing and giving reasons for his procedure to the surgeons eager for instruction, who streamed to him from all sides, and answering clearly all their questions. So certainly no one left King's College Hospital at that time without being a grateful and enthusiastic adherent of Lister's. What I however specially learnt beside the procedure, only carried out in its entirety by Lister, was that a special quality was necessary to the accomplishment of great things besides a fine intellect. People of ten call this quality pedantry - a designation which has a somewhat disdainful savour about it. I should rather describe it as precision and consistency. This purely personal trait was not only requisite for the discovery of the antiseptic treatment of wounds; it is to-day indispensable for every surgeon in his method of wound treatment, whether he calls it aseptic or antiseptic. If this quality is wanting to any one he will remain, in spite of all ability, a bungler in the treatment of wounds.

Yet I have already let myself venture too far on the subject. which space forbids my treating of to-day. It would be tempting to trace the wider development and transformation of the Listerian antisepsis to the still more finished asepsis 
of to-day, and in this way to bring into prominence how many of the original Listerian ideas, for example, the significance of air infection of wounds, have recently come into acceptation again after a long reign of errors. Unfortunately, I must not allow myself to venture on this.

Suture of the Patella.

The modern treatment of fractured patella, as is well known, not only leads us back to Lister because it is based on the antiseptic treatment of wounds, but because Lister was the first, just twenty-five years ago, to accomplish a regular suture of the patella. The first publication on the subject appeared in this JourNaL. "Six years later Lister was able to report 7 cases on which he had operated wich excellent results at the London Medical Society, and so the new method was definitely introduced into surgery. Countless surgeons of all nations-Howe, Chauvel. Telegnier, Schneider, Macewen, and Turner, Von Bergmann, Hackenbruch, Doebbelin, and Rossihave interested themselves in the extension and development of the procedure. The older operations for fractured patella as Malgaigne's hooks, subcutaneous suture of the patella as well as the treatment with different kinds of bandages and appliances were by this means expelled from the armamentarium of surgery; there was a time when the word "Lister" stood for right, and it was the duty of every one who was quite certain of antisepsis to treat fractured patella by suture. In course of time this view underwent limitation from the treatment of fractures involving joints by means of early massage and medico-mechanical methods. It was specially $\mathrm{Zam}$ Busch, who in a series of cases of fractured patella treated by medico-mechanical methods begun on the second or third day obtained excellent functional results. These observations were certainly weighty, for they brought to the remembrance of other surgeons their experience-one not unknown to Lister-that not uncommonly fractured pateilae recover with excellent results as to function even without bony union of the fragments. Without doubt early medicomeehanical treatment of fractured patellae by massage and active and passive movements is an excellent method, not only after the performance of suture, to promote restoration of function, but in many cases it re-establishes function even without suture. But it would certainly be a mistake to advance the treatment as routine practice for all fractured patellae, for a great number of these, even with the best medico-mechanical treatment, recover with poor or actually bad results. According to our present-day experience, it depends on our recognizing as a matter of course in respect of every case of fractured patella, whether it can unite with useful results without suture of the bone, or whether a surgical operation is necessary-a position held to-day by a great number of surgeons.

My communication shall be a contribution to the practical solution of this question from my own experience based on the final results which can be controlled in a precise way by the Roentgen-ray photograph. I give shortly the following nformation about 45 cases of fractured patella which I have observed in the last twelve years in the course of $\mathrm{my}$ practice at the Breslau Hospital.

With the greater number of the patients subsequent investigation could be made one to eleven years after the injury. Sixteen cases were treated purely medico-mechanically without operation. In 29 cases suture of the bones was carried out (in one case twice on account of a refracture).

Cases Suitable for Suture.

For deciding whether in an individual case suture of the fragment should be performed or not, the same principles generally and in the main prevailed as for the technical performance of the operation; for, founded on experience obtained in the course of twelve years many alterations have taken place, so that an imperfect agreement exists as to the technique of the operation and the indications for it in the reported cases. A certain inconsistency arose in this way from the fact that during my absence the assistants acting for me occasionally deviated slightly from my principles.

As the question is primarily whether in individual cases sutures of the bones shall be undertaken or not, I will touch in the first place on the existence of possible contraindications; for instance, suture of the bones was once omitted because the patient suffered at the same time from severe nephritis. On the basis of the positive indications which have swayed the choice of treatment I must speak further.

It is well known that fractures of the patella are divided into those which occur from direct violence arising from knocks and blows and those brought about by indirect violence-for example, from the wrenching apart of the patella as a result of a powerful contraction of the quadriceps. I will designate them shortly as "blow" and "tear" fractures. Views as to the proportion per cent. which one kind of fracture bears to the other are not yet elear. It appears, at all events, that pure tear fractures are not so frequent as was formerly thought to be the case ; anyhow, there exists a third possibility, and according to my experience this occurs very irequently - namely, a combination of a tear and blow fracture. If any one falls with a bent knee, it easily comes to pass that he powerfully contracts his quadriceps in order to save the fall or to right himself, and at the same time the patella suffers a blow from striking the ground or a projecting corner. It is plain that, through the combination of both these mechanical factors, fracture more easily occurs than as the result of only one of them. We should therefore have to distinguish between blow, tear, and combination fractures. For practical purposes, however, it is a matter of indifference whether we are concerned with a pure tear or with a combination fracture.

The difference between these two forms and a blow fracture is that in the last only the patella is broken, it matters not into how many pieces or in what direction. The chief thing is that with blow fractures the accessory ligaments remain attached to both sides of the patella. These strands of fibres, not very powerful in and for themselves, we may name them the parapatellar ligaments, reach out so as to prevent a wide separation of the fragments of the patella. They prevent the strong retraction of the quadriceps and maintain the continuity between the quadriceps tendon and the ligamentum proprium patellae. Now even if bony union does not take place between the fragments of the patella, yet the membranous strip of scar tissue connecting them remains short, and, in consequence of this, so strong that, with adequate medico-mechanical treatment, in its functional relations it becomes exactly, or almost exactly, like a patella in which bony nnion has occurred. For we must keep firm hold of the fact that experience shows that in nonoperative treatment only a strong connexion between the quadriceps tendon and the ligamentum patellae, such as is given by a membranous strip of scar tiseue, not bony union, is necessary for the restoration of a useful joint.

In contrast with ipure blow fractures are tear and combination fractures in which not only is the patella broken but the parapatellar ligaments are torn arart. In this way the continuity of the quadriceps tendon is quite interrupted and the fragments are displaced further and further from each other. The result as to function can then never be a good one for only a long feebly resisting scar forms the connecting link.

The decision as to whether in individual cases a pure blow fracture or a tear or especially a combination fracture exists may be frequently guessed on the ground of loss of consciousness, yet it is not at all a criterion in a critical examination of cases. Neither is the amount of separation decisive by itself. The only criterion is the behaviour of the quadriceps as regards function. If the patient soon after the injury is able to straighten the knee even with the help of a second person, this naturally is a fact in favour of the maintenance of continuity.

Nevertheless, all patients in whom the continuity of the quadriceps tendon is preserved may not be able to accomplish this because they avold a powerful contraction of the quadriceps on account of the pain. If on the other hand when one asks a patient suffering from an accident of this kind to attempt, at least, a movement of extension, one observes that the contracting quadriceps plainly draws up the upper fragment and so perceptibly increases the separation, this very widening of the gap from attempted extension shows a complete solution of continuity. In the same way in a similar case an increase in the gap occurs if the knee-joint is passively flexed.

If one carefully looks out for the alleged symptoms one prill 
easily form the judgement in tear or blow fracture, and then one can decide whether or not suture of the patella is necessary. Starting from this principle, I have with few exceptions abstained from suturing the patella in blow fractures, and limited myself exclusively to medico-mechanical treatment, whereas in combination and tear fractures suturing of the bones was carried out.

But I wish to note that I dissent decidedly from those who advise waiting for a time to see how function re-establishes itself. The decision must be made at the latest by the end of the first week, for the later one operates the more difficult, as is well known, does the operation become in its technical details and the more uncertain is the result.

With regard to the medico-mechanical treatment of fractured patella I can be concise, for its principles are known everywhere. Immobilization of the joint for a short time with elastic compression to hasten reabsorption of the effused blood, massage of the knee-joint, especially the quadriceps, starting from the second to the fourth day; the patient gets up at the end of the first week with a removable plaster-of-paris splint, which he lays aside at the end of the third week. At this period careful active and passive movements begin, and finally exercises with medico-mechanical apparatus.

The Operation of Suturing.

With regard to the suture of the bones, I should like to quote shortly the following details. I operate without exsanguinating the limb. I make the incision, as Lister originally suggested, in a transverse direction, corresponding to the line of fracture, and of course long enough not only to expose the patella, but also the torn parapatellar ligaments and the torn part of the capsule. This incision is decidedly to be preferred to the longitudinal incision recommended by other surgeons because of the clear view it gives of the parapatellar ligaments. For the suture $I$ formerly had recourse in the main to silver wire, latterly to the much stronger brass wire. When using brass wire no sublimate solution can be used, for it would render the wire brittle. I place through the patella-according to the length of the line of fracturethree or four sutures, which should not include the cartilage. I lay great stress on the fact that the accessory ligaments should be sutured on each side with one or two sutures as well as the patella. In this way the seriousness of a subsequent refracture of the patella is much lessened. According to the method, five to eight sutures in all will be applied in uniting the patella. Throughout the whole operation only asepsis is practised. The blood clot is removed with a sharp spoon during irrigation with sterile boiled salt solution. The wound is not drained, but completely closed; only $1 \mathrm{~cm}$. at each end of the skin incision remains unsutured. The gap yawning slightly or not at all is quite sufficient to allow for the escape of any subsequent oozing, and does not by so doing prevent healing by first intention. The after-treatment of sutured cases is in the main the same as of the unsutured, only we immobilize the limb during the healing of the wound - that is, for the first eight or ten days, so that medicomechanical treatment begins about a week later.

The foregoing refers chiefly to early operations-that is, those cases which come under our treatment forthwith after the injury. With later operations, in which the patient came under treatment as long as six months after the accident, we almost always had great difficulty in pulling down suffi ciently the upper fragment of the patella, strongly retracted by the shortened quadriceps. In most cases the tension of the muscle was abolished by making a V-shaped incision in the region of the rectus and cuneus, not in the tendon, which is converted into a $Y$ by pulling down the lower ends ; the continuity of the muscle was restored by sewing up the edges of the cut in the muscle with strong catgut sutures.

Results.

In subsequent investigations of our patients we examine first the result of healing in its anatomical bearings, that is, the kind of union between the fragments of the patella; in this connexion the $x$-ray picture gives the best information as to the degree of restitution, then the functional result. The goodness of this was judged with regard to three points: (I) According to the capacity for active flexion and extension; (2) the state of nutri- tion : of the quadriceps which affords the best index of the real condition of the part; (3) the power of the patient to go up steps and pursue his usual occupation. Corresponding to this we class those as good results in which a normal or nearly normal amount of function has returned: as moderaie results those in which the power of movement is somewhat limited and the capacity for heavy work lost, but nevertheless the power of moving on level ground is unimpaired. A noticeable limitation of movement or stiffness or impeded walking on level ground would be regarded as an unfavourable result.

Of 45 cases 16 were treated without operation; of these 15 were recent and $I$ an old fracture. In the last (it was a combination fracture) no operation was performed, in spite of the existing separation, on account of gevere nephritis, as has been previously stated. Capacity for use in this case remained continuously bad; the patient died three and a-quarter years after the accident. Of the 15 remaining cases, in 3 a combination and in 12 a pure blow fracture existed. We were able to observe 7 cases subsequently; in 2 further cases we received news by letter. In 8 instances the result was good, in I moderate. In the last case, even after six and a half years, according to the account in the letter, weakness and fatigue of the leg existed. I must note in addition that in this case there was a combination fracture. According to the principles by which I abide to-day, I should have operated on the case. A second case of combination fracture showed a good result as to function ten years after the injury in spite of a separation of $2 \frac{1}{2} \mathrm{~cm}$.

Of the 7 subsequently-investigated cases, in 5 the patella appeared on palpation to be firmly united. In 3 cases the $x$-ray picture showed a fissure at the line of fracture, while in the other 2 the line of fracture could no longer be recognized.

Suture of the bones was done 30 times for 29 patientstwice for one patient in consequence of refracture. In 21 instances they were recent fractures, which were operated on within the first week; 9 times they were old fractures. Of the recent fractures, 17 were tear or combination fractures, 4 pure blow fractures. The latter were operated on in spite of the continuity of the quadriceps tendon being maintained by the parapatellar ligaments, because of the considerable separation of the fragments. The occupation of the patients, which demanded restoration as complete as possible to normal conditions, was also a consideration which weighed with us.

Of the 30 cases, the operation wound healed by first intention with no trouble in 26 . In 4 instances healing was delayed by infection, and of these 2 were cases of late operation. I must here note that the 4 cases of infection all occurred in the first year of my practice at the Breslau Hospital before I had instituted the strict asepsis practised by me to-day. The 17 cases operated on in the last six and a-half years have healed without exception by first intention without the slightest interruption.

If the subsequent results of operation are in question, the following facts should be noted: Of the 17 cases of early operation, we could investigate 14 subsequently; 3 gave sufficient written information. The result was in 12 instances good, in 4 moderate, in I unsatisfactory. In the last case the sutures had cut through, and a separation of $1 \frac{1}{2}$ to $3 \mathrm{~cm}$. was left. A second case in which the sutures likewise tore through later, and a separation of $2 \mathrm{~cm}$. existed, contrasts with this one. In spite of all, the result as to function was good.

In the 14 cases subsequently investigated, in 12 the patella appeared to the touch to be closely united, but only in 8 cases did the $x$-ray picture show complete restoration in the anatomical sense, while in 4 crses a small cleft was visible. I have already mentioned that in 2 of these 14 cases a gap existed in consequence of the springing of the sutures.

Of the 9 cases of late suture we could subsequently investigate 7. In one we obtained news by letter. The functional result was good in 3 cases, 4 times moderate, once unsatisfactory. In the last case, as an examination of the nerves showed, a hysterical paralysis of the muscles of the right leg, especially those of the calf, was responsible for the bad result. The patella was firmly united. Of the 7 cases subsequently examined, the patella was firmly united in 6 instances, but the $x$ rays only showed bony union twice. In I a $\mathrm{g}$ ip of $\mathrm{I} \mathrm{cm}$. 
remained, but, notwithstanding this, the final result was good.

From the observations quoted we gather that even after medico-mechanical treatment, as well as after suture of the bones, the final result was, with few exceptions, very gratifying. The one unsatisfactory result of non-operative treatment was traceable to a wrong reading of indications, since, as I have already stated, acccrding to my present principles, suture of the patella should have been performed.

Of the 17 cases of primary suture of the patella of which the final result was known, only I showed a bad resplt. The reason of this was the cutting through of the suture, with considerable separation of the fragments. But neither can this unsatisfactory result be laid to the charge of the method as such, for by a reputed suture which was neglected a better result would doubtless have been obtained. Moreover, in this case the technique was so far deficient that the suture was carried out with catgut by one of my assistants contrary to our usual principles.

The 8 cases of late suture of the patella also gave satisfactory results with one exception. In the unfavourable case, however, a traumatic neurosis with hysterical paralysis lay at the root of the bad result; another circumstance for which the mode of operation cannot be made answerable.

We see, then, that both methods-the operative and the non-operative-give equally good results with a right interpretation of indications and a correct technique. These methods do no stand in opposition to each other as alternatives, but each supplements the other, and has it own definite, sharply. marked-off indications.

Dr. Schmidt, the assistant physician of my hospital, will give a detailed account of our results, with reproductions of the $x$-ray photographs, in Bruns's Beiträgen zur klinischen Chirurgie.

1 Lister, A New Operation for Fracture of the Patella, BRITISH Medical JOURNAL, 1877 , vol. ii, p. 850 .

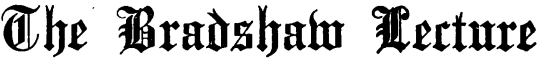

$$
\text { ON }
$$

\section{INFECTIVE ARTHRITIS.}

Delivered before the Royal College of Surgeons on December 10th,

\author{
BY HOWARD MARSH, F.R.C.S., \\ Surgeon to St. Bartholomew's Hospital.
}

Mr. President, Ladies and Gentlemen,-In my first words I must convey to you, Mr. President, my thanks for conferring upon me the honour of giving the Bradshaw Lecture this year. Within the walls of the Royal College of Surgeons of England, where Hunter laid the foundation and considerably advanced the development of the grandest museum of its kind in the world, where his spirit has ever since been present to inspire his followers; and where his work has been continued by Cooper, Lawrence, Owen, Paget, and our present distinguished curator, Professor Stewart; where our library grows year by year larger, and, as we believe, more and more useful for study and reference; and where at our examinations we have in the name of English surgery highly important duties to discharge, to be invited to lecture is an honour which he who is worthy of it cannot but warmly appreciate. Nor certainly for a moment can he forget the responsibilities which the office involves.

\section{LORD LISTER.}

But before I pass to the lecture itself, there is a circumstance to which Mr. President I think you would wish me to allude. On December 9 th, 1852 , just fifty years ago yesterday, a certain candidate passed his examination and became a Fellow of the College. He came from Essex, and his name was Joseph Lister.

What that name has since become, not only in the annals of English surgery, but among the greatest benefactors of the human race we all remember with admiration and with gratitude. The poet Gray alludes in trenchant phrase to those who shut the gates of mercy on mankind. Lord Lister has done more than any living man to throw them open. No matter in what century the roll of fame is called, the name of Lister can never henceforth be omitted from it.

Lord lister was never the man to seek recognition or reward, yet honours have flowed in upon him from every direction, and surely his cup became full when a few months ago he stood by and saw his principles turned to account in one of the most dramatic incidents of history, and when by appealing to them, Sir Frederick Treves, with conspicuous skill and conspicuous fortitude, averted the imminent peril which beset our King. The profound joy with which his subjects in every part of the world hailed the King's recovery was, in our profession, intensified by the fact that His Majesty, by constant devotion and earnest labour in the development of his Hospital Fund-which reached the unprecedented sum this year of $£ 100,000$-has made himself the greatest and most productive philanthropist of his generation.

Historical.

This lecture, together with a similar one at the Royal College of Physicians, was founded by the late Mrs. Bradshaw, to honour and perpetuate the memory of her husband the late Dr. William Wood Bradshaw, M.A., D.C.L.Oxford. Her action was at once affectionate and enlightened. She hoped that successive lecturers would, each in his turn, do something to advance the profession to the pursuit of which her husband had devoted his life. And it remains to each Bradshaw lecturer to give effect, to the best of his capacity and knowledge, to the objects which she had in view.

The subject I have chosen is that of Infective Arthritis ; in other words, I propose to discuss those diseases of the joints which are due to infection. I have not taken this subject as one that is altogether new, for it has long been known that in such instances as septicaemia, pyaemia-and other allied conditions - the joints are liable to become infected. I have chosen it because recent advances in bacteriology and exact clinical observation have made it clear that joint affections of this origin are much more frequent than was formerly supposed. Thus while no doubt much will still be added to our knowledge, the time, I think, has come when it may be useful briefly to review what has thus far been done.

In general pathology one of the main advances recently made has consisted in the discovery of the large part which infection by various micro-organisms plays in the production of disease. Thus, for example, peritonitis, only a few years ago, was supposed to arise as an independent form of inflammation, to be, as the phase was, idiopathic. It is now well established that no such form is ever met with, but that inflammation is, in the large proportion of cases, infective and due to microorganisms, of which several forms have been recognized. And as it is with the peritoneum, so it is with the joints. When inflammation occurs in a joint, while it is never idiopathic, it is often infective, and is produced, moreover, by agencies which, when they were 'first discovered, were supposed to be limited in their action to other structures. Indeed, there exists a close parallel between the peritoneum and the joints in regard to infection; and that this should be the case appears even at first sight probable when the similarity of structure between the peritoneum and the synovial mem. branes is borne in mind. Both consist of an epithelial layer and a substratum rich in blood vessels along which infective agents easily pass, and through the walls of which their migration can readily take place. A notable illustration of joint infection is met with in the case of the pneumococcus, or the bacillus lanceolatus. This micro-organism, originally discovered in the saliva and shown to be a pathogenic agent by Sternberg, was subsequently proved by Fraenkel to be the active agent in the production of acute lobar pneumonia, and, in the absence of any suspicion that it invaded other organs, it was termed the pneumococcus or lung coccus. Later investigations have shown, however, that it has a far wider range, and that it not only produces pleurisy, peritonitis, pericarditis, and meningitis, but also acute primary arthritis.

In fact, the general statement may now be made that in, at all events, the great majority of specific diseases, the joints are liable to infection. In compiling the list of these diseases tuberculosis and eyphilis need not now be considered, and the common forms of septicaemia and gonococcal infection are 80 well known that the need not be further alluded to. There will then remain the following: pneumococcal infection, 